\title{
Hybrid Cotangent Bundle Reduction of Simple Hybrid Mechanical Systems with Symmetry
}

\author{
Aaron D. Ames and Shankar Sastry \\ Department of Electrical Engineering and Computer Sciences \\ University of California at Berkeley \\ Berkeley, CA 94720 \\ \{adames, sastry\}@eecs.berkeley.edu
}

\begin{abstract}
This paper begins by introducing the notion of a simple hybrid mechanical system, which generalizes mechanical systems to include unilateral constraints on the configuration space. From such a system we obtain, explicitly, a simple hybrid system. The main contribution of this paper is to provide conditions on when it is possible to reduce the phase space of hybrid systems obtained from simple hybrid mechanical systems, and general simple hybrid systems, due to symmetries in the systems. Specifically, given a Hamiltonian G-spacewhich is the ingredient needed to reduce continuous systemswe find conditions on the hybrid system and the G-space so that reduction can be carried out in a hybrid setting-conditions that are explicitly related to conditions on the original hybrid mechanical system.
\end{abstract}

\section{INTRODUCTION}

Simple mechanical systems describe a very large class of physical systems since each consists of a configuration space $Q$ and a Hamiltonian $H: T^{*} Q \rightarrow \mathbb{R}$ of the form:

$$
H(q, p)=\frac{1}{2}\|p\|_{q}^{2}+V(q)
$$

where $T^{*} Q$ is the phase space, $\frac{1}{2}\|p\|_{q}^{2}$ is the kinetic energy and $V(q)$ is the potential energy. It is possible to reduce the dimensionality of systems of this form when they have symmetries through a process known as cotangent bundle reduction - one can reduce the dimensionality of the phase space (by "dividing" out by the symmetry group) and define a corresponding Hamiltonian on this reduced phase space. The main result of geometric reduction is that we can understand the behavior of the full-order system in terms of the behavior of the reduced system and vice versa. Therefore, the reduction of mechanical systems with symmetries plays a fundamental role in understanding the many important and interesting properties of these systems.

In this paper, we consider a class of mechanical systems with constraints (usually physical in nature) on the configuration space, i.e., there is a function $h: Q \rightarrow \mathbb{R}$ describing the admissible configurations of the system: $\left.Q\right|_{\{h(q) \geq 0\}}$. These systems are denoted by $\mathbf{H}$, and termed simple hybrid mechanical systems (HMS's). The term "hybrid" is used because the constraints on the configuration space result in discontinuities in the vector field describing the evolution of the mechanical system. Therefore, we can explicitly associate

\footnotetext{
*This research is supported by the National Science Foundation (NSF award number CCR-0225610)
}

a hybrid system, $\mathscr{H}_{\mathbf{H}}$, to a HMS. The main result of this paper is conditions on when it is possible to reduce hybrid systems of this form, and more general simple hybrid systems (hybrid systems with one domain and reset map).

We begin by considering a Hamiltonian G-space and give conditions on the elements of this G-space so that it defines a hybrid Hamiltonian G-space. Explicitly, this involves defining the notion of a hybrid group action and a hybrid momentum map, which is first done in the general setting of simple hybrid systems, followed by the special case of HMS's. Using these general notions, conditions are obtained on when a simple hybrid system, $\mathscr{H}$, can be reduced; the result is a simple hybrid system $\mathscr{H}_{\mu}$. Moreover, as with classical reduction, we can understand the behavior of $\mathscr{H}_{\mu}$ in terms of the behavior of $\mathscr{H}$-hybrid flows of $\mathscr{H}$ project to hybrid flows of $\mathscr{H}_{\mu}$.

Simple hybrid mechanical systems have been well-studied in the literature under many names (cf. [3] and the more than 1000 references therein), although the authors are unaware of any results regarding the reduction of systems of this form. Hybrid systems also have been well-studied in a geometric context, e.g., [4] studies hybrid mechanical systems and [6] considers hybrid systems with symmetries. The authors again are unaware of any results regarding the reduction of these systems, except for [2] which considers Routhian reduction with a focus on calculation.

Although never explicitly mentioned, the literature on classical reduction (cf. [1], [8], [9] and [10]) has touched upon issues relating to hybrid reduction. In [8] a form of discrete reduction is considered with assumptions very similar to conditions enumerated later in this paper. Similarly, the reduction of continuous systems with constraints has been studied in [7] and related references therein. Therefore, this paper can be viewed as the next logical step in understanding how to reduce the dimensionality of systems with symmetry.

\section{Simple HyBRID SySTEMS}

In this section, we introduce simple hybrid mechanical systems (HMS's) and simple hybrid systems-explicitly demonstrating how to associate a simple hybrid system to a HMS. This association is achieved through the use of Newtonian impact equations, which provide a method for describing the behavior of a mechanical system undergoing 
impacts, and results in a specific type of simple hybrid system, termed a Hamiltonian hybrid system.

Hamiltonians. The starting point for simple mechanical systems is a configuration space $Q$. Let $T Q$ be the tangent bundle of $Q$ and let $T^{*} Q$ be the cotangent bundle (the phase space). We denote the pairing between the vector spaces ${ }^{1}$ $T_{q}^{*} Q$ and $T_{q} Q$ by $\langle\cdot, \cdot\rangle: T_{q}^{*} Q \times T_{q} Q \rightarrow \mathbb{R}$, which for $(p, v) \in T_{q}^{*} Q \times T_{q} Q$ is given by $\langle p, v\rangle=\sum_{i=1}^{\operatorname{dim}(Q)} p_{i} v^{i}$.

Let $M(q)$ be the inertial matrix for a mechanical system and $K(q)=M(q)^{-1}$. For each $q \in Q$, we consider the $K(q)$-inner product on the vector space $T_{q}^{*} Q$ given by

$$
\left\langle\left\langle p, p^{\prime}\right\rangle\right\rangle_{q}=\sum_{i, j=1}^{\operatorname{dim}(Q)} p_{i} p_{j}^{\prime} K_{i j}(q),
$$

for $p, p^{\prime} \in T_{q}^{*} Q$; we use $\|\cdot\|_{q}$ to denote the corresponding norm on $T_{q}^{*} Q$. This induces (or is obtained from, depending on the perspective taken) an inner product on $T_{q} Q$ (the $M(q)$-inner product, which defines a Riemannian metric on $Q)$ via the Legendre transformation: $\mathbb{F} L: T Q \rightarrow T^{*} Q$, where $\mathbb{F} L(q, \dot{q})=(q, M(q) \dot{q})$.

A Hamiltonian is a map $H: T^{*} Q \rightarrow \mathbb{R}$. For this paper, we suppose that the Hamiltonian $H$ describes a mechanical system, i.e., that it has the following form ${ }^{2}$

$$
H(q, p)=\frac{1}{2}\|p\|_{q}^{2}+V(q),
$$

where $\frac{1}{2}\|p\|_{q}^{2}$ is the kinetic energy and $V(q)$ is the potential energy.

The cotangent bundle, $T^{*} Q$, is a symplectic manifold with its symplectic structure given by the canonical symplectic form

$$
\omega_{\text {canonical }}=\sum_{i=1}^{n} d q^{i} \wedge d p_{i} .
$$

With this symplectic form, we obtain a vector field on $T^{*} Q$ from a Hamiltonian, $X_{H}: T^{*} Q \rightarrow T\left(T^{*} Q\right)$, by requiring that it satisfies

$$
d(H)=\iota_{X_{H}} \omega_{\text {canonical }} .
$$

In coordinates, this yields the classical Hamiltonian equations

$$
\left(\begin{array}{c}
\dot{q} \\
\dot{p}
\end{array}\right)=X_{H}(q, p)=\left(\begin{array}{c}
\frac{\partial H}{\partial p}(q, p) \\
-\frac{\partial H}{\partial q}(q, p)
\end{array}\right) .
$$

We refer the reader to [1] and [9] for more details.

Definition 1: A simple hybrid mechanical system (HMS) is defined to be a tuple:

$$
\mathbf{H}=(Q, H, h),
$$

\footnotetext{
${ }^{1}$ We later will use the same notation to denote the pairing between a Lie algebra and its dual, as is common [8].

${ }^{2}$ Note that this Hamiltonian is obtained from a Lagrangian of the familiar form

$$
L(q, \dot{q})=\frac{1}{2} \dot{q}^{T} M(q) \dot{q}-V(q) .
$$
}

via the Legendre transformation. where $H$ is defined as in (1), and $h: Q \rightarrow \mathbb{R}$ defines constraints on the configuration of the system.

Definition 2: A simple hybrid system (so named because of its connection with simple HMS's, coupled with its "simple" structure) is a tuple:

$$
\mathscr{H}=(D, S, R, X),
$$

where

- $D$ is a smooth manifold called the domain,

- $S$ is an embedded submanifold of $D$ called the switching surface (or guard),

- $R$ is a smooth embedding $R: S \rightarrow D$ called the reset map (or state transition map),

- $X$ is a vector field on $D$.

We define a hybrid manifold as a tuple $\boldsymbol{D}^{\mathscr{H}}=(D, S, R)$ with elements as defined above. It is often useful to visualize a hybrid manifold in the form of a diagram:

$$
D \stackrel{\imath}{\longrightarrow} S \stackrel{R}{\longrightarrow} D,
$$

where $\imath$ is the natural inclusion.

We say that $\mathscr{H}=(D, S, R, X)$ is a Hamiltonian hybrid system with respect to a Hamiltonian $H$ if there exists a symplectic form $\omega$ on $D$ such that $(D, \omega, X)$ is a Hamiltonian system with respect to the Hamiltonian $H$, i.e., $d(H)=\iota_{X} \omega$.

The importance of this definition is that from a hybrid mechanical system, $\mathbf{H}=(Q, H, h)$, we can obtain a hybrid system, $\mathscr{H}_{\mathbf{H}}$, just as a dynamical system can be obtained from a classical simple mechanical system. This is done in two phases: first we define a hybrid manifold from $\mathbf{H}$ followed by the definition of $\mathscr{H}_{\mathbf{H}}$. Note that the hybrid system obtained from a hybrid Hamiltonian via the following construction has support in the literature (cf. [3], [4], [5]).

Hybrid Manifolds from HMS's. In order to construct a hybrid system from a HMS, we begin by contructing the hybrid manfold $\boldsymbol{D}_{\mathbf{H}}^{\mathscr{H}}=\left(D_{\mathbf{H}}, S_{\mathbf{H}}, R_{\mathbf{H}}\right)$ from a HMS $\mathbf{H}=$ $(Q, H, h)$. First, $D_{\mathbf{H}}$ and $S_{\mathbf{H}}$ are given as follows:

$$
\begin{aligned}
D_{\mathbf{H}} & =\left\{(q, p) \in T^{*} Q: h(q) \geq 0\right\}, \\
S_{\mathbf{H}} & =\left\{(q, p) \in T^{*} Q: h(q)=0 \text { and }\left\langle\left\langle p, d h_{q}\right\rangle\right\rangle_{q} \leq 0\right\} .
\end{aligned}
$$

This is exactly the setup in mechanical systems with unilatural constraints. With this in mind, we can define a state transition map $R_{\mathbf{H}}$ by

$$
R_{\mathbf{H}}(q, p)=\left(q, P_{q}(p)\right)
$$

where $P_{q}: T_{q}^{*} Q \rightarrow T_{q}^{*} Q$ is given by

$$
P_{q}(p)=p-(1+e) \frac{\left\langle\left\langle p, d h_{q}\right\rangle\right\rangle_{q}}{\left\|d h_{q}\right\|_{q}^{2}} d h_{q},
$$

with $0 \leq e \leq 1$ is the coefficient of restitution, e.g., for a perfectly elastic impact $e=1$, and for a perfectly plastic impact $e=0$.

Hybrid Systems from HMS's. Since we already have constructed a hybrid manifold $\boldsymbol{D}_{\mathbf{H}}^{\mathscr{H}}=\left(D_{\mathbf{H}}, S_{\mathbf{H}}, R_{\mathbf{H}}\right)$ from 


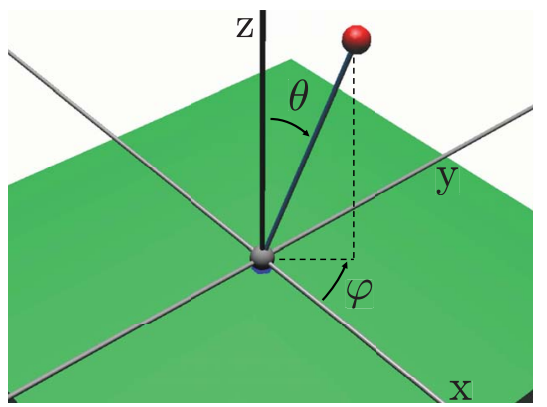

Fig. 1. Spherical pendulum mounted on the floor as given in the running example.

the hybrid mechanical system $\mathbf{H}$, it only remains to define the vector field $X_{\mathbf{H}}$. Using the canonical symplectic form, $\omega_{\text {canonical }}$, we define $X_{\mathbf{H}}=X_{H}$, as given in (2). Finally, $\mathscr{H}_{\mathbf{H}}=\left(D_{\mathbf{H}}, S_{\mathbf{H}}, R_{\mathbf{H}}, X_{\mathbf{H}}\right)$. It trivially follows that $\mathscr{H}_{\mathbf{H}}$ is a Hamiltonian hybrid system w.r.t. the Hamiltonian $H$.

Example 1: The running example in this paper will be a spherical pendulum mounted on the floor (Fig. 1). Here $Q_{\mathbf{P}}=\mathbb{S}^{2}$ and, using the standard spherical coordinates, we denote an element $q \in \mathbb{S}^{2}$ by $q=(\theta, \varphi)$ and we denote an element $p \in T_{q}^{*} \mathbb{S}^{2}$ by $p=\left(p_{\theta}, p_{\varphi}\right)$. For this example, the Hamiltonian $H_{\mathbf{P}}$ is given by

$$
H_{\mathbf{P}}(q, p)=\frac{1}{2 m R^{2}}\left(p_{\theta}^{2}+\frac{p_{\varphi}^{2}}{\sin ^{2}(\theta)}\right)-m g R \cos (\theta) .
$$

Finally, $h_{\mathbf{P}}$ is the height function $h_{\mathbf{P}}(\theta, \varphi)=R \cos (\theta)$, i.e., we have a simple hybrid mechanical system given by $\mathbf{P}=$ $\left(Q_{\mathbf{P}}, H_{\mathbf{P}}, h_{\mathbf{P}}\right)$.

The hybrid manifold for the spherical pendulum $D_{\mathrm{P}}^{\mathscr{P}}=$ $\left(D_{\mathbf{P}}, S_{\mathbf{P}}, R_{\mathbf{P}}\right)$ is given by

$$
\begin{aligned}
D_{\mathbf{P}} & =\left\{\left(\theta, \varphi, p_{\theta}, p_{\varphi}\right) \in T^{*} \mathbb{S}^{2}: \cos (\theta) \geq 0\right\}, \\
S_{\mathbf{P}} & =\left\{\left(\theta, \varphi, p_{\theta}, p_{\varphi}\right) \in T^{*} \mathbb{S}^{2}: \cos (\theta)=0 \text { and } p_{\theta} \geq 0\right\},
\end{aligned}
$$

and

$$
R_{\mathbf{P}}\left(\theta, \varphi, p_{\theta}, p_{\varphi}\right)=\left(\theta, \varphi,-e p_{\theta}, p_{\varphi}\right) .
$$

Finally, the vector field is given by

$$
X_{\mathbf{P}}(q, p)=\left(\begin{array}{c}
\frac{p_{\theta}}{m_{\varphi} R^{2}} \\
\frac{-p_{\varphi}^{2}}{m R^{2} \sin ^{2}(\theta)} \\
\frac{m R^{2} \cos (\theta) \sin ^{2}(\theta)}{0}-m g R \sin (\theta)
\end{array}\right),
$$

and $\mathscr{P}=\left(D_{\mathbf{P}}, S_{\mathbf{P}}, R_{\mathbf{P}}, X_{\mathbf{P}}\right)$.

Hybrid flows. A hybrid flow is a tuple:

$$
\chi^{\mathscr{H}}=(\Lambda, \mathcal{J}, \mathcal{C})
$$

where

- $\Lambda=\{0,1,2, \ldots\} \subseteq \mathbb{N}$ is a finite or infinite indexing set,

- $\mathcal{J}=\left\{I_{i}\right\}_{i \in \Lambda}$ is a hybrid interval where $I_{i}=\left[\tau_{i}, \tau_{i+1}\right]$ if $i, i+1 \in \Lambda$ and $I_{N-1}=\left[\tau_{N-1}, \tau_{N}\right]$ or $\left[\tau_{N-1}, \tau_{N}\right)$ or
$\left[\tau_{N-1}, \infty\right)$ if $|\Lambda|=N, N$ finite, with $\tau_{i}, \tau_{i+1}, \tau_{N} \in \mathbb{R}$ and $\tau_{i} \leq \tau_{i+1}$,

- $\mathcal{C}=\left\{c_{i}\right\}_{i \in \Lambda}$ is a collection of solutions of $X$, i.e., $\dot{c}_{i}(t)=X\left(c_{i}(t)\right)$ for all $i \in \Lambda$,

such that the following conditions hold for every $i, i+1 \in \Lambda$,

$$
\begin{array}{cc}
\text { (i) } & c_{i}\left(\tau_{i+1}\right) \in S \\
\text { (ii) } & R\left(c_{i}\left(\tau_{i+1}\right)\right)=c_{i+1}\left(\tau_{i+1}\right) .
\end{array}
$$

The initial condition for the hybrid flow is $x_{0}=c_{0}\left(\tau_{0}\right)$. When we wish to make explicit the initial condition of $\chi^{\mathscr{H}}$, we write $\chi^{\mathscr{H}}\left(x_{0}\right)$.

\section{HyBRID HAMILTONIAN G-SPACES}

The purpose of this section is to introduce the notion of a hybrid Hamiltonian G-space, the starting point for which is a Hamiltonian G-space with respect to the continuous portion of $\mathscr{H}$. We discuss hybrid Hamiltonian G-spaces in the context of both simple hybrid systems and HMS's; in the later case, explicit constructions are carried out.

\section{A. General Case: Simple Hybrid Systems}

Hamiltonian G-spaces. The starting point for reduction is a Hamiltonian G-space (cf. [1], [9], [10]) which is a tuple:

$$
(D, \omega, \Phi, J),
$$

where

- $(D, \omega)$ is a symplectic manifold,

- $\Phi$ is a symplectic action of a Lie group $G$ on $D$, i.e., $\Phi: G \times D \rightarrow D$ and $\Phi_{g}^{*} \omega=\omega$ for all $g \in G$,

- $J: D \rightarrow \mathfrak{g}^{*}$ is an $\mathrm{Ad}^{*}$-equivariant momentum mapping for this action.

The question is: what is the hybrid version of this data? More specifically, what is the hybrid version of a Lie group action? A hybrid momentum map?

Hybrid group actions. Let $\mathscr{H}=(D, S, R, X)$ be a hybrid system. Consider an action $\Phi: G \times D \rightarrow D$ of a Lie group $G$ on $D$. We say that this is a hybrid action if $\left.\Phi_{g}\right|_{S}$ is an action of $G$ on $S$ and for all $g \in G$ :

$$
\left.R \circ \Phi_{g}\right|_{S}=\Phi_{g} \circ R \text {. }
$$

That is, for all $g \in G$ we have a commuting diagram:

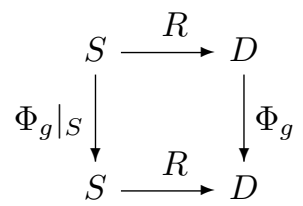

Or, in other words, $R$ is equivariant with respect to the actions $\Phi$ and $\left.\Phi\right|_{S}$. We say that $\Phi$ is a free and proper hybrid action, if $\Phi$ is a free and proper action that is hybrid.

Hybrid orbit spaces. For the hybrid manifold $D^{\mathscr{H}}=$ $(D, S, R)$, a Lie group $G$, and $\Phi$ a hybrid action, we define the hybrid orbit space as a tuple:

$$
\boldsymbol{D}^{\mathscr{H}} / G=(D / G, S / G, \widehat{R}),
$$


where $D / G$ and $S / G$ are the orbit spaces of $\Phi$ and $\left.\Phi\right|_{S}$, respectively, and $\widehat{R}: D / G \rightarrow S / G$ is the induced map.

We would like to give conditions on the hybrid action $\Phi$ so that $\boldsymbol{D}^{\mathscr{H}} / G$ is a hybrid manifold, i.e., such that we have a diagram

$$
D / G \stackrel{\imath}{\hookleftarrow} S / G \stackrel{\widehat{R}}{\rightarrow} D / G
$$

in which $D / G$ is a smooth manifold, $S / G$ is an embedded submanifold and $\widehat{R}$ is a smooth map. In fact, conditions for when these occur are well-known (cf. [1]).

Proposition 1: If $\Phi: G \times D \rightarrow D$ is free and proper hybrid action, then $\boldsymbol{D}^{\mathscr{H}} / G$ is a hybrid manifold. Moreover, there is a submersion $\pi: D \rightarrow D / G$ such that the following diagram

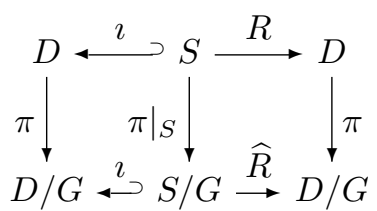

commutes and $\left.\pi\right|_{S}$ is a submersion.

Definition 3: An $\mathrm{Ad}^{*}$-equivariant momentum map $J$ is said to be a hybrid $\mathrm{Ad}^{*}$-equivariant momentum map if the following diagram

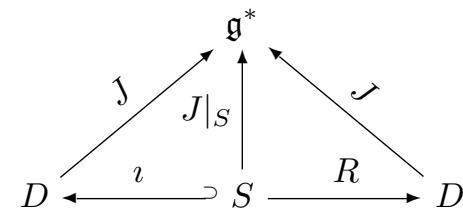

commutes.

Definition 4: A hybrid Hamiltonian G-space is a tuple

$$
\left(\boldsymbol{D}^{\mathscr{H}}, \omega, \Phi, J\right),
$$

such that $(D, \omega)$ is a symplectic manifold, $\Phi$ is a hybrid symplectic action, and $J$ is a hybrid $\mathrm{Ad}^{*}$-equivariant momentum map.

\section{B. Special Case: Simple Hybrid Mechanical Systems}

Lifted group actions. For a hybrid mechanical system, $\mathbf{H}=$ $(Q, H, h), D_{\mathbf{H}}=\left.T^{*} Q\right|_{\{h(q) \geq 0\}}$. Therefore, it is natural to consider actions on $T^{*} Q$ that are obtained by lifting an action on $Q$. Specifically, for an action $\Psi: G \times Q \rightarrow Q$, we obtain an action of $G$ on $T^{*} Q$ by cotangent lifts, i.e., we obtain an action $\Psi^{T^{*}}: G \times T^{*} Q \rightarrow T^{*} Q$ by defining

$$
\Psi^{T^{*}}(g,(q, p)):=T^{*} \Psi_{g^{-1}}(q, p)=\left(\Psi_{g}(q), \Psi_{g^{-1}}^{*}(p)\right) .
$$

It is possible to give conditions on when this action is a hybrid action by considering the constraint function $h$, the potential energy $V$, and $H$.

Definition 5: A simple hybrid mechanical system $\mathbf{H}=$ $(Q, H, h)$ is said to be G-invariant if there is an action $\Psi$ of $G$ on $Q$ such that $h$, the potential energy $V$, and $H$ are G-invariant:

$$
\begin{gathered}
h\left(\Psi_{g}(q)\right)=h(q), \quad V\left(\Psi_{g}(q)\right)=V(q), \\
H\left(\Psi_{g}^{T^{*}}(q, p)\right)=H(q, p),
\end{gathered}
$$

for all $g \in G$; note that the last assumption says that $H$ is G-invariant under the lifted action, which holds iff

$$
\left\langle\left\langle\Psi_{g^{-1}}^{*}(\cdot), \Psi_{g^{-1}}^{*}(\cdot)\right\rangle\right\rangle_{\Psi_{g}(q)}=\langle\langle\cdot, \cdot\rangle\rangle_{q},
$$

when coupled with the assumption on the G-invariance of the potential energy.

Proposition 2: If $\mathbf{H}=(Q, H, h)$ is $G$-invariant, then the lifted action $\Psi^{T^{*}}$ of $G$ on $D$ is a hybrid action.

Momentum maps for HMS's. For simple mechanical systems, there is an explicit definition of an $\mathrm{Ad}^{*}$-equivariant momentum map $J_{\mathbf{H}}$. Let $\Psi$ be the action of $G$ on $Q$, and define a vector field on $Q$ by

$$
\xi_{Q}(q)=\left.\frac{d}{d t} \Psi(\exp (t \xi), q)\right|_{t=0} \in T_{q} Q
$$

for $\xi \in T_{e} G \cong \mathfrak{g}$. Using this, we can define $J_{\mathbf{H}}$ and prove that it is a hybrid $\mathrm{Ad}^{*}$-equivariant momentum map under easily verifiable conditions.

Proposition 3: For $\mathbf{H}=(Q, H, h)$, if $h$ is G-invariant, then $J_{\mathbf{H}}: D_{\mathbf{H}} \subseteq T^{*} Q \rightarrow \mathfrak{g}^{*}$ defined by

$$
\left\langle J_{\mathbf{H}}(q, p), \xi\right\rangle=\left\langle p, \xi_{Q}(q)\right\rangle,
$$

is a hybrid Ad*-equivariant momentum map.

Combining the results from Propositions 2 and 3, we have the following theorem that provides easily verifiable conditions on when a specific Hamiltonian G-space associated to a HMS is a hybrid Hamiltonian G-space.

Theorem 1: If $\mathbf{H}=(Q, H, h)$ is G-invariant, then

$$
\left(\boldsymbol{D}_{\mathbf{H}}^{\mathscr{H}}, \omega, \Psi^{T^{*}}, J_{\mathbf{H}}\right)
$$

is a hybrid Hamiltonian G-space.

Example 2: For our running example, $G_{\mathbf{P}}=\mathbb{S}^{1}$, which acts by rotations about the vertical axis, i.e., $\Psi_{\mathbf{P}}: \mathbb{S}^{1} \times Q_{\mathbf{P}} \rightarrow$ $Q_{\mathbf{P}}$ is given by

$$
\Psi_{\mathbf{P}}(\psi,(\theta, \varphi))=(\theta, \varphi+\psi),
$$

and the lifted action on $D_{\mathbf{P}}$ is given by

$$
\Psi_{\mathbf{P}}^{T^{*}}\left(\psi,\left(\theta, \varphi, p_{\theta}, p_{\varphi}\right)\right)=\left(\theta, \varphi+\psi, p_{\theta}, p_{\varphi}\right),
$$

which is clearly a hybrid action by Proposition 2. Now for $\xi \in \mathfrak{g}_{\mathbf{P}} \cong \mathbb{R}$,

$$
\xi_{Q_{\mathbf{P}}}(\theta, \varphi)=(0, \xi) \in T_{(\theta, \varphi)} Q_{\mathbf{P}},
$$

so the momentum map is given by

$$
J_{\mathbf{P}}\left(\theta, \varphi, p_{\theta}, p_{\varphi}\right)=p_{\varphi},
$$


which is a hybrid momentum map by Proposition 3. Finally, it follows from Theorem 1 that

$$
\left(\boldsymbol{D}_{\mathbf{P}}^{\mathscr{P}}, \omega_{\text {canonical }}, \Psi_{\mathbf{P}}^{T^{*}}, J_{\mathbf{P}}\right)
$$

is a hybrid Hamiltonian G-space.

\section{HYBRID SySTEM REDUCTION}

We begin by reviewing the classic phase space reduction theorem, first proven by Marsden and Weinstein [10]. We use this theorem to prove the existence of a reduced Hamiltonian hybrid system given a Hamiltonian hybrid system together with hybrid Hamiltonian G-space. Moreover, we are able to prove a relationship between the hybrid flows of these two systems - a result that is very similar to the classical trajectory reduction theorem.

The reduced phase space. Let $(D, \omega, \Phi, J)$ be a Hamiltonian G-space, and assume that $\mu \in \mathfrak{g}^{*}$ is a regular value of $J$. If

$$
G_{\mu}=\left\{g \in G: \operatorname{Ad}_{g^{-1}}^{*} \mu=\mu\right\}
$$

is the isotropy subgroup of $G$, then the action $\Phi$ of $G$ on $D$ restricts to an action of $G_{\mu}$ on $J^{-1}(\mu)$,

$$
\Phi: G_{\mu} \times J^{-1}(\mu) \rightarrow J^{-1}(\mu)
$$

because of the $\mathrm{Ad}^{*}$-equivariance of $J$. Moreover, if the action of $G_{\mu}$ on $J^{-1}(\mu)$ is free and proper, then $D_{\mu}=$ $J^{-1}(\mu) / G_{\mu}$ is a manifold, referred to as the reduced phase space, and there is a submersion $\pi_{\mu}: J^{-1}(\mu) \rightarrow D_{\mu}$. Finally, the main theorem of [10] says that $D_{\mu}$ has a unique symplectic form $\omega_{\mu}$ with the property:

$$
\pi_{\mu}^{*} \omega_{\mu}=\imath_{\mu}^{*} \omega
$$

where $\imath_{\mu}: J^{-1}(\mu) \rightarrow D$ is the inclusion.

Hybrid regular values. Let $\mathscr{H}=(D, S, R, X)$ be a hybrid system. Suppose that $\mu$ is a regular value of $J: D \rightarrow \mathfrak{g}^{*}$. We say that this is a hybrid regular value if it is also a regular value of $\left.J\right|_{S}$. This implies, when coupled with the commuting diagram (6), that the following diagram

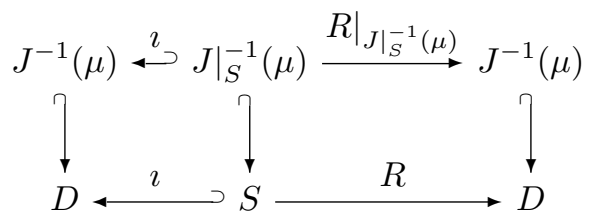

commutes, where $J^{-1}(\mu)$ and $\left.J\right|_{S} ^{-1}(\mu)$ are embedded submanifolds.

Theorem 2: Let $\left(\boldsymbol{D}^{\mathscr{H}}, \omega, \Phi, J\right)$ be a hybrid Hamiltonian $G$-space. Assume $\mu \in \mathfrak{g}^{*}$ is a hybrid regular value of a hybrid $\mathrm{Ad}^{*}$-equivariant momentum map $J$ and that the action of $G_{\mu}$ on $J^{-1}(\mu)$ is free, proper and hybrid. Then

$$
\begin{aligned}
\boldsymbol{D}_{\mu}^{\mathscr{H}} & =\left(D_{\mu}, S_{\mu}, R_{\mu}\right) \\
& :=\left(J^{-1}(\mu) / G_{\mu},\left.J\right|_{S} ^{-1}(\mu) / G_{\mu}, \widehat{\left.R\right|_{\left.J\right|_{S} ^{-1}(\mu)}}\right)
\end{aligned}
$$

is a hybrid manifold.

The reduced hybrid phase space. The hybrid manifold introduced in the above theorem is referred to as the reduced hybrid phase space. To better understand this hybrid manifold, note that the submersion $\pi_{\mu}$ together with (6) and (9) yields the following commuting diagram

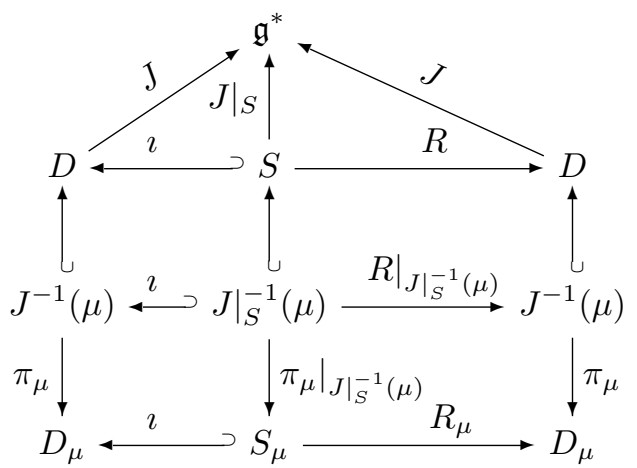

where $\left.\pi_{\mu}\right|_{\left.J\right|_{S} ^{-1}(\mu)}$ is also a submersion; this implies that $R_{\mu}$ is defined by requiring that the bottom right square in this diagram commute.

Theorem 3: Given a Hamiltonian hybrid system $\mathscr{H}=$ $(D, S, R, X)$ w.r.t. a G-invariant Hamiltonian $H$, and an associated hybrid Hamiltonian G-space satisfying the assumptions of Theorem 2, then there is a reduced Hamiltonian hybrid system (w.r.t. $H_{\mu}$ )

$$
\mathscr{H}_{\mu}=\left(D_{\mu}, S_{\mu}, R_{\mu}, X_{\mu}\right),
$$

where $D_{\mu}^{\mathscr{H}}=\left(D_{\mu}, S_{\mu}, R_{\mu}\right)$ is defined as in Theorem 2, and $X_{\mu}$ is defined by $d\left(H_{\mu}\right)=\iota_{X_{\mu}} \omega_{\mu}$.

Reduced Hamiltonians. If $H$ is a $G$-invariant Hamiltonian on $D$, then the reduced Hamiltonian $H_{\mu}$ on $D_{\mu}$ is defined uniquely by requiring that

$$
H_{\mu} \circ \pi_{\mu}=H \circ \imath_{\mu} .
$$

If $\left(D, \omega, X_{H}\right)$ is a Hamiltonian system for the Hamiltonian $H$, then the classical reduction theorem says that there is an associated reduced Hamiltonian system $\left(D_{\mu}, \omega_{\mu}, X_{H_{\mu}}\right)$ for the Hamiltonian $H_{\mu}$. These two Hamiltonian Systems are related to each other in the following way: if $c(t)$ is the flow of $X_{H}$ with initial condition $c\left(t_{0}\right) \in J^{-1}(\mu)$, then $\pi_{\mu}(c(t))$ is the flow of $X_{H_{\mu}}$ with initial condition $\pi_{\mu}\left(c\left(t_{0}\right)\right)$. The hybrid analogue of this is given in the following theorem.

Theorem 4: With $\mathscr{H}$ and $\mathscr{H}_{\mu}$ as in Theorem 3, if $\chi^{\mathscr{H}}\left(x_{0}\right)$ is a hybrid flow of $\mathscr{H}$ with $x_{0} \in J^{-1}(\mu)$, then there is a corresponding hybrid flow $\chi^{\mathscr{H}_{\mu}}$ of $\mathscr{H}_{\mu}$ defined by

$$
\chi^{\mathscr{H}_{\mu}}\left(\pi_{\mu}\left(x_{0}\right)\right)=\left(\Lambda, \mathcal{J}, \pi_{\mu}(\mathcal{C})\right)
$$

where $\pi_{\mu}(\mathcal{C}):=\left\{\pi_{\mu}\left(c_{i}\right): c_{i} \in \mathcal{C}\right\}$.

The hybrid reduction result given in Theorem 3 only provides, to quote [11], "soft" information about the reduced theorem in that it does not yield a method for explicitly 

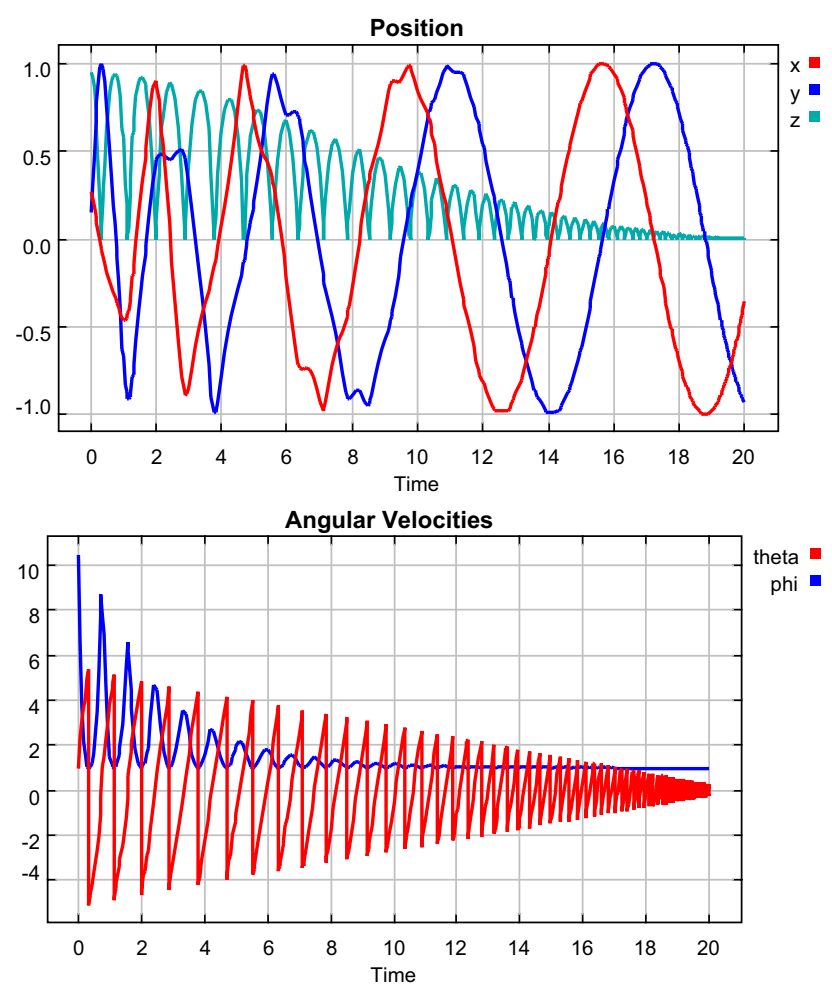

Fig. 2. Reconstruction of the reduced spherical pendulum: position of the mass over time (top), angular velocities over time (bottom).

constructing the reduced Hamiltonian hybrid system. There are more concrete methods for computing the reduced system by using methods from classical mechanics which allow for the explicit reduction of Hamiltonians (see [1], [8], [11]). This can be generalized in a rather straight-forward manner to HMS's when they are $G$-invariant. The end result is two methods for reducing a hybrid system associated to a HMS, described graphically by:

$$
\begin{aligned}
& \mathbf{H} \stackrel{\text { association }}{\longrightarrow} \mathscr{H}_{\mathbf{H}} \stackrel{\text { reduction }}{\longrightarrow}\left(\mathscr{H}_{\mathbf{H}}\right)_{\mu} \\
& \mathbf{H} \stackrel{\text { reduction }}{\longrightarrow} \mathbf{H}_{\mu} \stackrel{\text { association }}{\longrightarrow} \mathscr{H}_{\mathbf{H}_{\mu}}
\end{aligned}
$$

It is possible to show that the processes of "association" and "reduction" commute, i.e., the order in which they are taken is irrelevant. This can be visualized in a commuting diagram of the form:

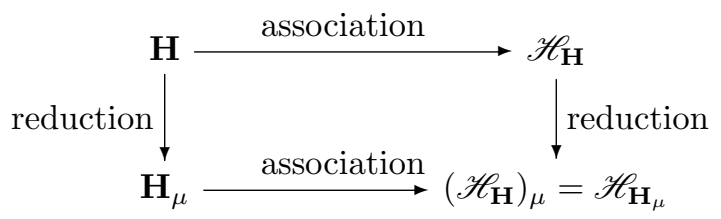

This result yields a method for computing reduced hybrid systems obtained from HMS's.

Example 3: Returning to the spherical pendulum mounted on the ground, we can explicitly calculate the reduced hybrid system for this example. We first compute the associated reduced HMS $\mathbf{P}_{\mu}=\left(Q_{\mathbf{P}_{\mu}}, H_{\mathbf{P}_{\mu}}, h_{\mathbf{P}_{\mu}}\right)$ and then associate to the system a simple hybrid system using the techniques outlined in Section II. The continuous portion of the reduction follows from [8]. In this example, $T^{*}\left(Q_{\mathbf{P}} / G_{\mathbf{P}}\right)$ is identified with $T^{*}\left(S^{1} / \mathbb{Z}_{2}\right)$, i.e., $Q_{\mathbf{P}_{\mu}}=S^{1} / \mathbb{Z}_{2}$. The reduced Hamiltonian $H_{\mathbf{P}_{\mu}}: T^{*}\left(Q_{\mathbf{P}_{\mu}}\right)=T^{*}\left(S^{1} / \mathbb{Z}_{2}\right) \rightarrow \mathbb{R}$ is given by

$$
H_{\mathbf{P}_{\mu}}\left(\theta, p_{\theta}\right)=\frac{1}{2} \frac{p_{\theta}^{2}}{m R^{2}}+m g R \cos (\theta)+\frac{1}{2} \frac{\mu^{2}}{m R^{2} \sin ^{2}(\theta)} .
$$

Finally, we have $h_{\mathbf{P}_{\mu}}(\theta)=R \cos (\theta)$.

The hybrid manifold for the reduced spherical pendulum $\boldsymbol{D}_{\mathbf{P}_{\mu}}^{\mathscr{P}}=\left(D_{\mathbf{P}_{\mu}}, S_{\mathbf{P}_{\mu}}, R_{\mathbf{P}_{\mu}}\right)$ is given by

$$
\begin{aligned}
D_{\mathbf{P}_{\mu}} & =\left\{\left(\theta, p_{\theta}\right) \in T^{*}\left(Q_{\mathbf{P}_{\mu}}\right): \cos (\theta) \geq 0\right\}, \\
S_{\mathbf{P}_{\mu}} & =\left\{\left(\theta, p_{\theta}\right) \in T^{*}\left(Q_{\mathbf{P}_{\mu}}\right): \cos (\theta)=0 \text { and } p_{\theta} \geq 0\right\},
\end{aligned}
$$

and

$$
R_{\mathbf{P}_{\mu}}\left(\theta, p_{\theta}\right)=\left(\theta,-e p_{\theta}\right) .
$$

Finally, the vector field is given by

$$
X_{\mathbf{P}_{\mu}}\left(\theta, p_{\theta}\right)=\left(\begin{array}{c}
\frac{p_{\theta}}{m R^{2}} \\
m g R \sin (\theta)+\frac{\mu^{2} \cos (\theta)}{m R^{2} \sin ^{3}(\theta)}
\end{array}\right)
$$

and $\mathscr{P}_{\mu}=\left(D_{\mathbf{P}_{\mu}}, S_{\mathbf{P}_{\mu}}, R_{\mathbf{P}_{\mu}}, X_{\mathbf{P}_{\mu}}\right)$. It can be verified by direct inspection that in fact this hybrid system is the reduced hybrid system associated to $\mathscr{P}$ as given in Theorem 3 because it makes the diagram in (10) commute.

Note that in this example it is easy to reconstruct the trajectories of the full-order pendulum from the reduced pendulum through integration. A trajectory of the full-order pendulum mounted on the ground, as reconstructed from the reduced system, can be found in Figure 2; here $e=.95$, $R=1$ and $m=1$. Note that both the full-order pendulum and the reduced pendulum are Zeno with these parameters.

\section{REFERENCES}

[1] R. Abraham and J. E. Marsden, Foundations of Mechanics. Benjamin/Cummings Publishing Company, 1978.

[2] A. D. Ames and S. Sastry, "Hybrid Routhian reduction of Lagrangian hybrid systems," in Proceedings of the 25th American Control Conference, Minneapolis, MN, 2006.

[3] B. Brogliato, Nonsmooth Mechanics. Springer-Verlag, 1999.

[4] F. Bullo and M. Žefran, "Modeling and controllability for a class of hybrid mechanical systems," IEEE Transactions on Automatic Control, vol. 18 , no. 4 , pp. 563-573, 2002.

[5] R. C. Fetecau, J. E. Marsden, M. Ortiz, and M. West, "Nonsmooth Lagrangian mechanics and variational collision integrators," SIAM J. Applied Dynamical Systems, vol. 2, pp. 381-416, 2003.

[6] J. Hu and S. Sastry, "Symmetry reduction of a class of hybrid systems," in Hybrid Systems: Computation and Control, ser. Lecture Notes in Computer Science, C. J. Tomlin and M. R. Greenstreet, Eds., vol. 2289. Springer-Verlag, 2002, pp. 267-280.

[7] C. M. Marle, "Reduction of constrained mechanical systems and stability of relative equilibria," Comm. Math. Phys., vol. 174, no. 2, pp. 295-318, 1995.

[8] J. E. Marsden, Lectures on Mechanics, ser. London Mathematical Society Lecture Note Series. Cambridge University Press, 1992, vol. 174.

[9] J. E. Marsden and T. S. Ratiu, Introduction to Mechanics and Symmetry, ser. Texts in Applied Mathematics. Springer, 1999, vol. 17.

[10] J. E. Marsden and A. Weinstein, "Reduction of symplectic manifolds with symmetry," Rep. Math. Phys., vol. 5, pp. 121-130, 1974.

[11] W. J. Satzer, "Canonical reduction of mechanical systems under abelian group actions with an application to celestial mechanics," Indiana Univ. Math. J., vol. 26, no. 5, pp. 951-976, 1977. 